\title{
EVALUATION OF RETENTION PROPERTIES OF TWO DIFFERENT STUD ATTACHMENTS IN MANDIBULAR IMPLANT OVERDENTURES
}

\author{
Nada Sherin El Khourazaty*
}

\begin{abstract}
Objectives: The aim of this study was to compare the retention values of two stud attachments with two different designs in Mandibular implant overdenture.

Materials \& methods: Eight completely edentulous patients were selected to receive mandibular complete overdentures supported by 4 implants. At the prosthetic phase the patients were divided into two groups; Group I: 4 Patients of this group received a mandibular overdenture retained by a ball abutment and a nylon matrix, Group II: 4 Patients of this group received a mandibular overdenture retained by OLS attachment with a PEEK matrix. Retention values were measured in vitro using universal testing machine at the time of installation, 6 and 12 months.

Results: The results of this study demonstrated no significant difference between the conventional ball with nylon cap and the PEEK attachment at the installation time, while analysis of the retentive values after 6 and 12 months showed a significant decrease in retention values of the ball and socket attachment with nylon matrix, while the OLS attachment with PEEK matrix showed higher retentive means compared to the ball.
\end{abstract}

Conclusion: Within the limitation of this study it was concluded that PEEK OLS attachment attains better values of retention with time when compared to conventional ball and socket attachments with nylon matrix.

\section{INTRODUCTION}

Mandibular complete overdenture treatment has been a valuable option for decades, but its use was limited when the treatment depended on retained teeth as overdenture abutments. This treatment, however, is currently experiencing more popularity than ever before. In fact, Dentistry may be experiencing a dramatic shift, in which mandibular implant overdenture treatment may become the new standard of care for the treatment of the edentulous mandible. Practitioners are looking for more simple treatment options that can provide cost effective alternatives to more complex implant prosthodontics procedures. Implant overdenture provide a strong return for the investment in treatment time and cost and are the treatment

"Lecturer of removable Prosthodontics, Faculty of Dentistry, Cairo University. 
suited to the lower socioeconomic status of many edentulous patients. The clinical outcome of this treatment is significantly better than that achieved with conventional mandibular dentures, especially when patients are experiencing technical problems because of compromised prosthesis retention or stability. ${ }^{1}$

Implant retained overdenture offers multiple advantages when compared to conventional removable dentures. Mostly, efficient retention and stability is easily achieved by a fixed restoration or by using overdenture's attachments instead of depending on the physical means of retention with conventional dentures. ${ }^{2}$

Convertibility is considered one of the major advantages of the overdentures, where a welldesigned complete denture could be easily converted into an implant stabilized prosthesis and since a fewer number of implants are needed in overdenture prosthesis, it was considered by many authors to be more economic. ${ }^{3}$

It is well known that retention is a key element in success of removable prosthodontics, as it greatly affects patient satisfaction. Patient complaints are mostly related to lack of retention and stability, both of which could be attributed to many factors related to the anatomy of the patients as well as denture construction. These factors could dramatically compromise the retention of the denture, especially the lower one. ${ }^{4-7}$

Therefore, many studies have recommended the mandibular implant-retained overdentures as an effective treatment modality for edentulous patients and, in particular, those who have persistent problems using conventional mandibular prosthesis. Placing from 2 to 4 implants with attachments can improve the retention and stability of dentures and can achieve greater support by projecting axial loads into the bone. ${ }^{8-10}$

Different types of attachments have been used for connecting the denture and the implants such as bar connectors, stud attachments, magnets, and rigid or resilient telescopic copings. However, the necessity for simplifying the selection and the use of attachments for overdenture fixation is very important, as there is no need for a highly complicated and expensive attachment when there is a less sophisticated type that can perform the same function with no additional expenses. ${ }^{11-13}$

Stud attachments are one of the most commonly used attachments with overdentures due to their ease of handling, cost effectiveness, less technique sensitivity, better stress distribution, and acceptable retention..$^{14,15}$

Retention of the stud attachment is variable and mainly dependant on the attachment design as well as the material of the matrix insert which might be polyoxymethelene, polyethylene, rubber, polycarbonate or nylon. ${ }^{16-18}$

Recently, Poly Ether Ether Ketone (PEEK) material was introduced as an ideal partner in prosthetic and implant dentistry due to its good mechanical and physical properties. PEEK has shown acceptable flexibility with high mechanical resistance to wear and high tensile, fatigue and flexural strength. PEEK is used to produce high-quality plastic parts that are thermo-stable and both electrically and thermally insulating. It also attains low specific mass, elasticity similar to the one of bone, and an almost non-existent material fatigue. ${ }^{19,20}$

\section{MATERIALS \&METHODS}

Eight male completely edentulous patients were selected from the outpatient clinic of Prosthodontics Department; Faculty of Oral and Dental Medicine, Cairo University. The patients' age ranged from fifty to sixty five years old and were systemically free from any disease that may interfere with dental implant placement and/or osseointegration, in addition have adequate bone for implant placement, as well as sufficient inter arch space for overdenture construction with normal maxillomandibular 
relation. Only cooperative patients, following instructions and having proper neuromuscular co-ordination were included in the study. Patient history, clinical examination and radiographic assessment were carefully carried out to verify the selection criteria.

The pre-surgical preparation required the construction of conventional upper and lower complete denture. If the patient's old denture were judged satisfactory regarding the tissue adaptation, occlusion and esthetics, construction of the new dentures was skipped.

The finished lower denture was duplicated and a radio opaque acrylic resin stent was done for each patient. Four small holes were drilled in the stent at the proposed implant sites and a cone beam CT image was obtained.

Four $3.7 \mathrm{~mm}$ diameter implants were placed with the aid of the surgical guide in the canine and molar region area using sequential drilling after flap reflection. The direction of drilling was kept perpendicular to the bone and midway buccolingually, putting in consideration the parallelism between the four implants. Three months following surgical implant installation, limited flap reflection was done for placing the abutments and patients were prepared for the prosthetic phase and randomly divided into two groups:

- Group I: 4 Patients of this group received a mandibular overdenture retained by a ball abutment and a nylon matrix*. Fig (1) \& Fig (2)

- Group II: 4 Patients of this group received a mandibular overdenture retained by OLS attachment with a PEEK matrix.** Fig (1) \& Fig (2)

The matrices and housings of both attachments were secured firmly over the stud abutments for both groups, Holes corresponding to the housings were opened in the fitting surface of the denture allowing seating of the denture without any interference with the housings, as proved by absence of rocking, pressure indicating paste and proper occlusion. Cold curing resin was placed in the relieved areas of the denture and the denture was seated in the patient mouth. The resin was left to polymerize while the patient was closing in centric jaw relation with gentle pressure .The overdenture was removed, trimmed and polished with the housings picked up in its fitting surface.
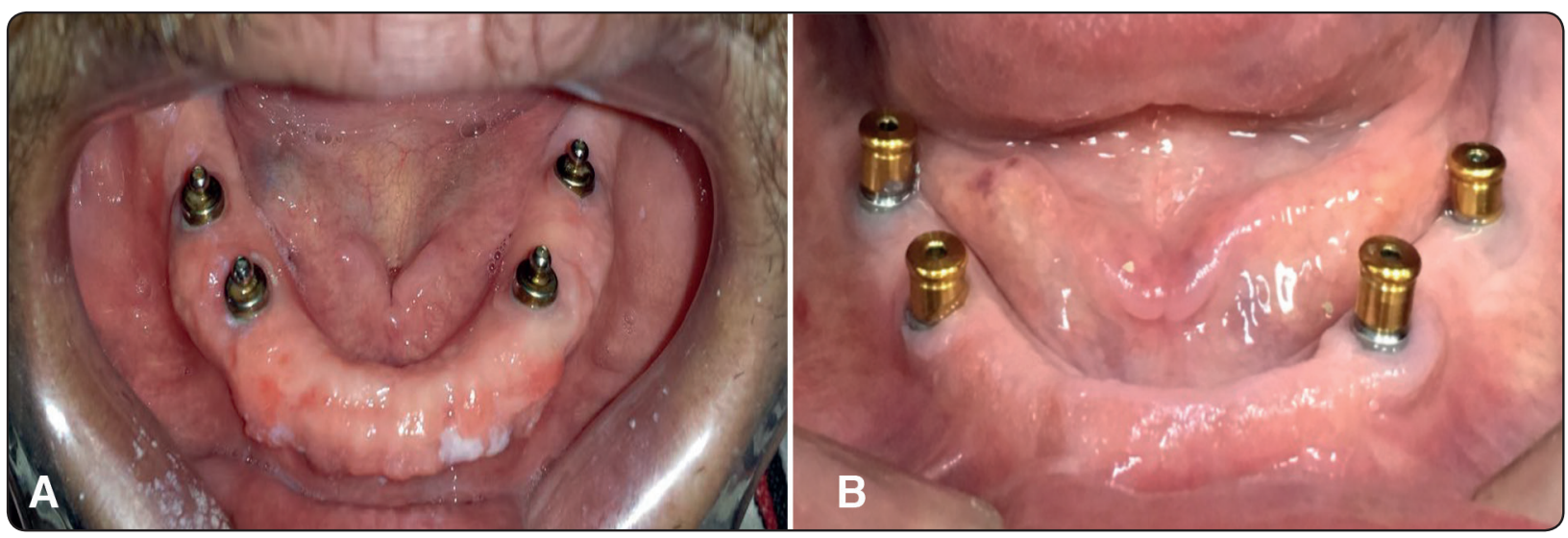

Fig. (1) A: Patient with ball and socket attachment. B: Patient with OLS attachment.

\footnotetext{
* Implant Direct Sybron International

** Osteoseal dental implants, California, USA
} 
After the direct attachment pick up was done, the attachments were removed from the patient's mouth and secured in its place in the denture then the fixture analogues were attached to them and a cast model was poured.

For retention measurement the relative geometric center was determined in order to place the hook attached to the universal testing machine. The geometric center of the mandibular denture was identified by connecting the anterior and posterior extremities of the denture (midline and retromolar pads respectively) to form a triangle, the intersection of the three lines bisecting the three angles of the triangle was considered as the geometric center. A wrought wire, $1 \mathrm{~mm}$ in diameter was bent at its center and adjusted so as to run two $\mathrm{cm}$ above the Occlusal Plane between the two retromolar pads. A second wrought wire, $1 \mathrm{~mm}$ in diameter was adjusted to extend from the lingual flange upwards till it meets the other wire at the predetermined geometric center and shaped to form a c-shaped hook around the first wire. The ends of the wires were then fixed to the polished surface of the lower denture by self-cured acrylic resin. Groove indices were done at the polished surface of the denture where the wires were attached to allow reattaching of the wires at the same position during testing

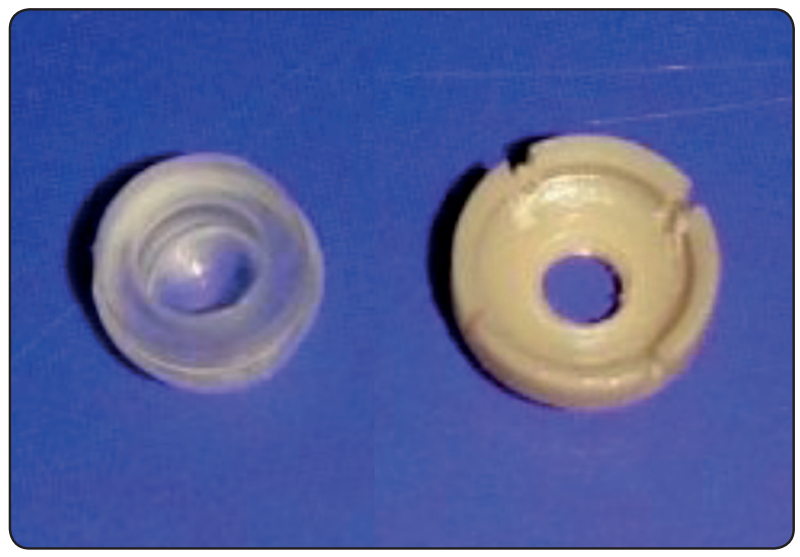

Figure (2): Right: Nylon Matrix, Left: PEEK Matrix with hole and slits

* NEXYGEN from Lloyd Instruments. the retention at different time intervals throughout the study. Retention was measured at the time of denture placement (initial retention), six and twelve months later.

The retention was evaluated using a Universal Testing Machine. ${ }^{*}$ Each cast with the corresponding implant supported overdenture was secured to the lower fixed compartment of the testing machine (Model LRX-plus; Lloyd Instruments Ltd., Fareham, UK) with a load cell of $5 \mathrm{kN}$ and data were recorded using computer software (Nexygen-MT Lloyd Instruments). The overdenture was suspended from the upper movable compartment of the testing machine by an orthodontic wire loop $(0.7 \mathrm{~mm})$ with the loading axis of machine. A tensile load with pull out mode of force was applied via materials testing machine at a crosshead speed of $5 \mathrm{~mm} / \mathrm{min}$ (Figure $3)$. The load required to dislodgment was recorded in Newton. Twenty reading were recorded for each denture in each group.

Finally, all the wrought wires were removed and denture was polished again and delivered to the patient. This procedure was repeated at 6 and 12 months intervals after installing the wrought wires with the hook which represent the geometrical center at the prepared indices at the polished denture surface.

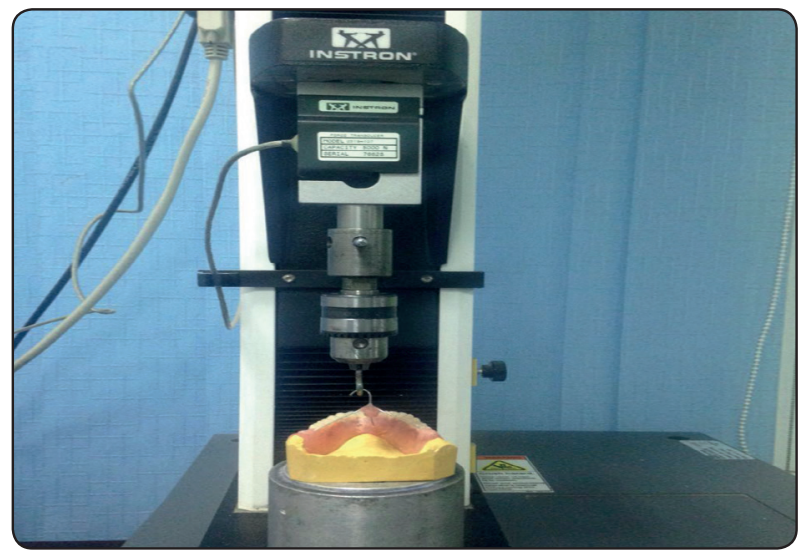

Fig. (3): Denture in place with attached wires forming a loop at the geometrical center and attached to the universal testing machine. 


\section{RESULTS}

This randomized control study aimed to evaluate the retention values of two different Stud attachments with two different matrix materials at the time of insertion (initial retention), 6 months and 12 months.

The mean and standard deviation values were calculated for each group. Data were explored for normality using Kolmogorov-Smirnov and Shapiro-Wilk tests and showed parametric (normal) distribution. Independent sample-t test was used to compare between independent samples for parametric data. The significance level was set at $\mathrm{P} \leq 0.05$. Statistical analysis was performed with IBM ${ }^{\circledR}$ SPSS $®$ Statistics Version 20 for Windows.

At the time of denture placement both attachments showed no statistically significant difference in mean of retention where $(p=0.213)$. The highest mean of retention was found in Ball \& Socket (75.36 $\pm 5.21)$ attachment while the least mean of retention was found in Peek OLS attachment (68.34 \pm 3.08$)$.

On the other hand when comparing the retention values of both attachments after 6 months, There was a statistically significant difference in mean of retention between Ball \& Socket and Peek OLS attachments where $(\mathrm{p}=0.001)$.The highest mean of retention was found in Peek OLS $(76.24 \pm 5.18)$ attachment while the least mean of retention was found in Ball \& Socket $(55.07 \pm 4.04)$ attachment.

Similar results were obtained when comparing the retention values for both attachments at 12 months as they showed A statistically significant difference in mean of retention where $(\mathrm{p}=0.003)$. The highest mean of retention was found in Peek OLS (35.25 \pm 3.90$)$ attachment while the least mean of retention was found in Ball \& Socket (20.50 \pm 0.06$)$ attachment.

TABLE (1) The mean, standard deviation (SD) values of retention along different times of both groups.

\begin{tabular}{|c|c|c|c|}
\hline \multirow{2}{*}{ Variables } & Initial measurements & $\mathbf{6}$ months & 12 months \\
\cline { 2 - 4 } & Mean \pm SD & Mean \pm SD & $20.50 \pm 0.06^{\mathrm{a}}$ \\
\hline Ball \& Socket & $75.36 \pm 5.21^{\mathrm{a}}$ & $45.07 \pm 4.04^{\mathrm{a}}$ & $35.25 \pm 3.90^{\mathrm{b}}$ \\
\hline Peek OLS & $70.34 \pm 3.08^{\mathrm{a}}$ & $56.24 \pm 5.18^{\mathrm{b}}$ & $\mathbf{0 . 0 0 3}^{*}$ \\
\hline $\boldsymbol{P}$-value & $\mathbf{0 . 2 1 3 n s}$ & $\mathbf{0 . 0 0 1 *}$ & $\mathbf{P}^{*}$ \\
\hline
\end{tabular}

Mean with different letters in the same column indicate statistically significance difference

*; significant $(p<0.05)$ ns; non-significant ( $p>0.05)$

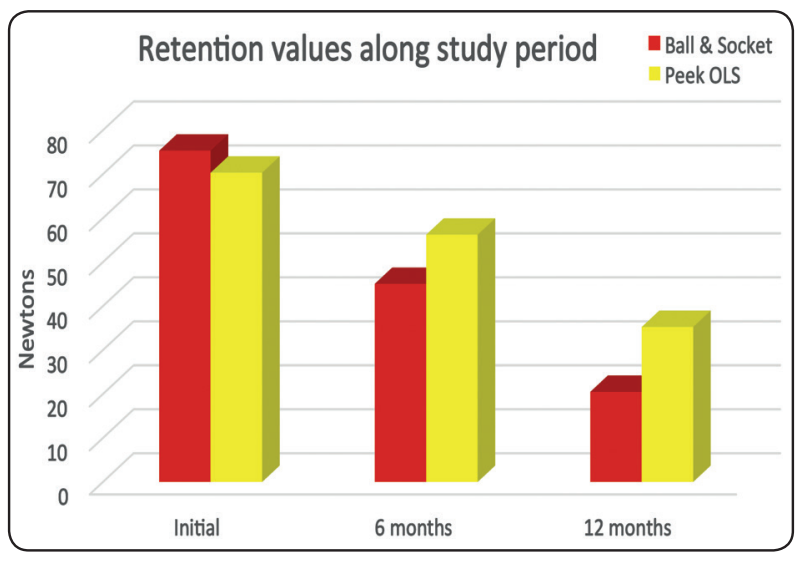

Fig. (4): Bar chart representing means of retention along different times of both groups.

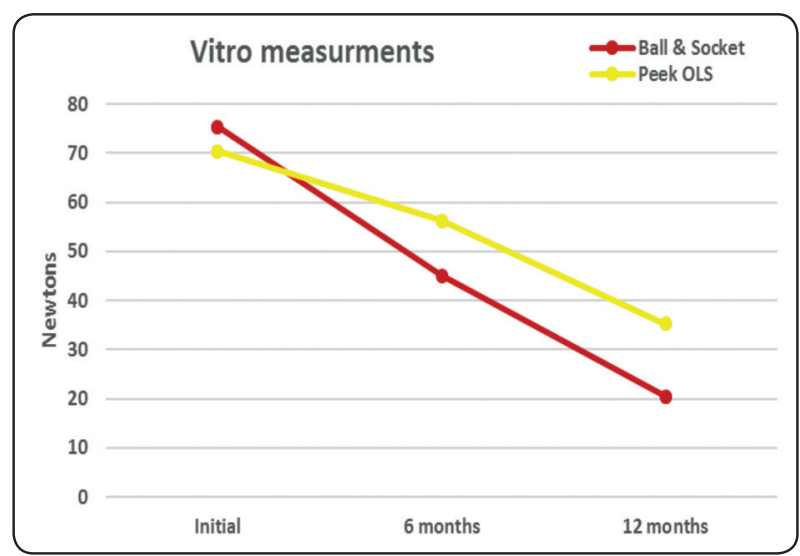

Fig. (5): Line chart representing means of retention along different times of both groups. 


\section{DISCUSSION}

The implant retained overdenture is a removable prosthesis obtaining its retention and support through an attachment, retention and resistance of the implant-tissue supported overdenture may be affected by the type of attachment ${ }^{21}$ as the choice of attachment system being directly associated with patient satisfaction and prosthetic prognosis, a wide selection of attachment systems is currently available; each possesses their respective advantages and disadvantages. Attachment selection should be made based on the dental arch shape, inter arch space, technique sensitivity, ease of adjustment, functional life, and retentive forces. ${ }^{22}$

Since there has been no clear consensus concerning the optimal retentive force of a denture, In addition to very limited amount of data regarding to the retentive force of the attachments provided by the manufacturers. The literature regarding the retentive force of the attachment system varies widely, even for similar types of attachment systems, depending on the study design as well as experimental conditions such as the direction of force application, and the distance and angle of attachments. ${ }^{12-15,23}$

Attachments for non-splinted implants are known for their susceptibility to wear and as an expendable part they have to be exchanged after a certain time of clinical service which directly disturbs the retentive value of the attachment. That is why changing the matrix of the attachment is considered as a frequent maintenance intervention in implant retained overdentures. ${ }^{24}$

There are limited studies on an attachment system with a retentive element made from PEEK are available. The results of this research demonstrated no significant difference between the PEEK attachment and the conventional ball with nylon cap at the installation time as both materials revealed clinically accepted results regarding retention force. On the other hand, analysis of the retentive values after 6 and 12 months show a significant decrease in retention values of the ball and socket attachment with nylon matrix, while the OLS attachment with PEEK matrix recorded higher retentive means compared to the ball. This might be directly attributed the fact that the wear of the nylon cap might be more obvious than the PEEK $^{19,24,25}$ additionally, the design of the OLS attachment attains longer parallel walls which could provide some friction forces that aid in retention. Finally, the improved retention behavior of the PEEK attachment in the present investigation can be explained by its design and material. The PEEK matrix provides a slot in the matrix and a hole on top, this slot and hole expand when connecting the matrix and the patrix and might act as a buffer, which might reduce the deterioration of the matrix surface resulting in a reduced wear of the material. ${ }^{19,25}$

\section{CONCLUSION}

Within the limitation of this study it was concluded that PEEK OLS attachment attains better values of retention when compared to conventional ball and socket attachments with nylon matrix. Additionally PEEK material can be used as a retentive cap for a stud attachment.

\section{REFERENCES}

1. Burns, D. R. The mandibular complete overdenture. Dental clinics of North America 48, 603-23, v-vi (2004).

2. Burns, D. R., Unger, J. W., Elswick, R. K. \& Beck, D. A. Prospective clinical evaluation of mandibular implant overdentures: Part I--Retention, stability, and tissue response. The Journal of prosthetic dentistry 73, 354-363 (1995).

3. Sadowsky, S. J. The implant-supported prosthesis for the edentulous arch: design considerations. The Journal of prosthetic dentistry 78, 28-33 (1997).

4. Kim, H.-Y., Lee, J.-Y., Shin, S.-W. \& Bryant, S. R. Attachment systems for mandibular implant overdentures: a systematic review. The journal of advanced prosthodontics 4, 197-203 (2012). 
5. Zarb G A, Bolender C L, Hickey J C, C. G. C. Boucher's Prosthodontic Treatment for Edentulous Patients. 10th edn. St. Louis: C.V.Mosby, 1-27 (1990). doi:10.1016/00223913(92)90474-O

6. Gosavi, S. S., Ghanchi, M., Malik, S. A. \& Sanyal, P. A Survey of Complete Denture Patients Experiencing Difficulties with their Prostheses. Journal of Contemporary Dental Practice 14, 524-527 (2013).

7. Huumonen, S. et al. Residual ridge resorption, lower denture stability and subjective complaints among edentulous individuals. Journal of Oral Rehabilitation 39, 384-390 (2012).

8. Tokuhisa, M., Matsushita, Y. \& Koyano, K. In vitro study of a mandibular implant overdenture retained with ball, magnet, or bar attachments: comparison of load transfer and denture stability. The International journal of prosthodontics 16, 128-134 (2003).

9. Svetlize, C. a \& Bodereau, E. F. Comparative study of retentive anchor systems for overdentures. Quintessence international (Berlin, Germany : 1985) 35, 443-448 (2004).

10. Boven, G.C., G.M. Raghoebar, A. Vissink, et al. Improving masticatory performance, bite force, nutritional state and patient's satisfaction with implant overdentures: a systematic review of the literature.No Title. J Oral Rehabil 42, 220-33 (2015).

11. Naert, I., Quirynen, M., Hooghe, M. \& van Steenberghe, D. A comparative prospective study of splinted and unsplinted Br??nemark implants in mandibular overdenture therapy: A preliminary report. The Journal of Prosthetic Dentistry 71, 486-492 (1994).

12. Besimo, C. E. \& Guarneri, A. In vitro retention force changes of prefabricated attachments for overdentures. Journal of Oral Rehabilitation 30, 671-678 (2003).

13. Krennmair, G., Weinländer, M., Krainhöfner, M. \& Piehslinger, E. Implant-supported mandibular overdentures retained with ball or telescopic crown attachments: a 3-year prospective study. The International journal of prosthodontics 19, 164-170 (2005).

14. Passia, N. et al. Single dental implant retained mandibular complete dentures--influence of the loading protocol: study protocol for a randomized controlled trial. Trials 15 , 186 (2014).
15. Rabbani, S., Juszczyk, A. S., Clark, R. K. F. \& Radford, D. R. Investigation of Retentive Force Reduction and Wear of the Locator Attachment System with Different Implant Angulations. Quintessence Puplishing Co, Ltd. 30, 556563 (2015).

16. Chung, K.-H., Whiting, D., Kronstrom, M., Chan, D. \& Wataha, J. Retentive characteristics of overdenture attachments during repeated dislodging and cyclic loading. The International journal of prosthodontics 24, 127-9 (2011).

17. Stergiou, a, Juszczyk, a S., Clark, R. K. F. \& Radford, D. $\mathrm{R}$. The retentive forces of the locator attachment system at different angulations. The European journal of prosthodontics and restorative dentistry 20, 168-74 (2012).

18. Schweyen, R., Beuer, F., Arnold, C. \& Hey, J. Retentive characteristics of a vinylpolysiloxane overdenture attachment system. Clinical Oral Investigations 19, $947-$ 953 (2015).

19. Passia, N., Ghazal, M. \& Kern, M. Long-term retention behaviour of resin matrix attachment systems for overdentures. Journal of the Mechanical Behavior of Biomedical Materials 57, 88-94 (2016).

20. Bayer, S. et al. Retention force of plastic clips on implant bars: A randomized controlled trial. Clinical Oral Implants Research 23, 1377-1384 (2012).

21. Tabatabaian, F., Alaie, F. \& Seyedan, K. Comparison of three attachments in implant-tissue supported overdentures: an in vitro study. Journal of dentistry (Tehran, Iran) 7, 113 8 (2010).

22. Davis, D. M. Role of implants in the treatment of edentulous patients. The International journal of prosthodontics 3, 4250 (1990).

23. Cakarer, S., Can, T., Yaltirik, M. \& Keskin, C. Complications associated with the ball, bar and locator attachments for implant-supported overdentures. Medicina Oral, Patologia Oral y Cirugia Bucal 16, (2011).

24. Andreiotelli, M., Att, W. \& Strub, J.-R. Prosthodontic complications with implant overdentures: a systematic literature review. The International journal of prosthodontics 23, 195-203 (2010).

25. Rutkunas, V., Mizutani, H., Takahashi, H. \& Iwasaki, N. Wear simulation effects on overdenture stud attachments. Dental Materials Journal 30, 845-853 (2011). 